\title{
The Lived Experiences of Patients with Allergic Rhinitis in a Referral Institution in the Ashanti Region of Ghana
}

\author{
Mercy Belins Beyuo ${ }^{1}$, Andrew Adjei Druye ${ }^{2}$, Rita Larsen-Reindorf ${ }^{3}$, Irene Korkoi Aboh ${ }^{2 *}$, Philomina \\ Woolley 4 , Issahalq Duah Mohammed ${ }^{5}$ and Anna Konney ${ }^{5}$
}

${ }^{1}$ Ear, Nose and Throat Nursing School, Adum-Kumasi, Ghana

${ }^{2}$ School of Nursing and Midwifery, University of Cape Coast, Cape Coast, Ghana

${ }^{3}$ Clinical Care Unit, Ghana Health Service, Ashanti Region, Ghana

${ }^{4}$ Nurses and Midwifery Council for Ghana, Accra, Ghana

${ }^{5}$ Ear Nose Throat Department, Komfo Anokye Teaching Hospital, Kumasi, Ghana

*Corresponding author: Irene Korkoi Aboh, School of Nursing and Midwifery, University of Cape Coast, Cape Coast, Ghana

\begin{abstract}
Aim: The aim of this study was to explore the experiences of patients with allergic rhinitis on quality of life.

Methods: The study used qualitative approach by purposively sampling patients diagnosed with Allergic Rhinitis at a referral center. A face-to-face interview was conducted using an interview guide with prompts and the data analyzed using the matic analysis.

Results: The findings of the study revealed that patients with allergic rhinitis experience several signs and symptoms once they come into contact with the triggers and this condition has negative effect on their physical and psychological wellbeing. Physically, the participants experienced excessive sneezing, itchy eyes, nose and throat, runny nose and blocked nose, headache and pain in the throat, coughing, fatigue, inability to smell as well as hawking of the throat. Psychologically, participants experienced sleep and emotional disturbances, labeling, anxiety and discomfort, strained relationship with family and poor concentration.
\end{abstract}

\section{Keywords}

Allergic rhinitis, Experiences, Institution, Patients, Wellbeing

\section{Introduction}

Allergic Rhinitis (AR) is a global health problem and an inflammatory disease of the nasal mucosa, its symptoms imposes a substantial burden [1]. It has the affinity to impair both the quality of life that impact on school, work, and leisure activities, as a result impairing patients'
Quality of Life (QoL) [2]. Its association with comorbidities like rhino-sinusitis, otitis media, pharyngitis, allergic conjunctivitis, nasal polyps and asthma is massive, meanwhile comorbid asthma is common in allergic rhinitis [3]. Allergic rhinitis is a public health problem with a prevalence of $10 \%$ to $40 \%$ and the trend affecting more than 400 million people worldwide, with high prevalence recorded in the developed countries [4]. Allergic Rhinitis is classified into seasonal and perennial rhinitis which focuses on its episodic nature [5]. But recently with its impact on Asthma (ARiA) it has been classified based on the duration and severity of the presenting manifestations into persistent and intermittent forms, which can be mild and moderate to severe [6]. Allergic inflammation does not limit itself to the nasal airway. Multiple co-morbidities have been associated with it [7]. Co-morbidities such as bronchial asthma is a chronic respiratory illness affecting the airways and making breathing difficult. These symptoms occur mainly due to inflammation and swelling of the airways with secretion of mucus which causes obstruction and promotes further breathing difficulty. Nasal congestion is identified as a common and frequent problem, while seasonality of symptoms was uncommon. Symptoms affected sleep of sufferers, and this was every night. Patients felt miserable due to allergic rhinitis even though they always followed instructions for taking rhinitis medication [8].

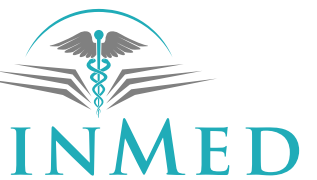

INTERNATIONAL LIBRARY

Citation: Beyuo MB, Druye AA, Reindorf RL, Aboh IK, Woolley P, et al. (2022) The Lived Experiences of Patients with Allergic Rhinitis in a Referral Institution in the Ashanti Region of Ghana. Int Arch Nurs Health Care 8:169. doi.org/10.23937/2469-5823/1510169

Accepted: January 17, 2022: Published: January 19, 2022

Copyright: (c) 2022 Beyuo MB, et al. This is an open-access article distributed under the terms of the Creative Commons Attribution License, which permits unrestricted use, distribution, and reproduction in any medium, provided the original author and source are credited. 
Quality of Life (QoL) is the psychological, physical and social functioning, which integrate with positive aspects of wellbeing as well as negative aspects of disease or infirmity [9]. The concept encompasses patients' subjective perception of the effect of the condition and its treatment on their daily life. The symptoms experienced by patients with allergic rhinitis can lead to both physical and psychological complications associated with decreased learning performances and attention disorders. Even though not life threatening, its symptoms are normally troublesome adversely affecting work and Quality of Life (QoL), and impose a substantial burden on both the individual and the society [10]. Symptoms such as sneezing, rhinorrhea, nasal congestion and nasal pruritus, ocular and palatal pruritus, redness and lacrimation, and postnasal drainage usually affects the physical wellbeing of patients [2]. Patients also experience negative effects on their psychological wellbeing, which include poor concentration, behavioural disorders, such as restlessness, irritability, inattention, and daytime sleepiness [11]. The aim of the study was to explore the lived experiences of patients with allergic rhinitis at a referral institution in the Ashanti Region of Ghana.

\section{Materials and Methods}

This study used descriptive qualitative approach because qualitative studies in health care, illness and nursing-related phenomena are best appreciated with a focus to bring out the lived experiences of participants and gaining insights into a poorly understood phenomenon. The studies targeted all patients who report at a referral center with allergic rhinitis with an impact on Asthma (ARiA) over a period of 42 days. The study was conducted at the only tertiary health facility in the geographical area that serves the middle zone of Ghana. It is a training center for all categories of skilled medical practitioners.

\section{Data collection}

Eligible participants for this study included patients diagnosed with Allergic Rhinitis aged 18 years and above. Patients who had obvious nasal pathology like nasal polyps, deviated nasal septum, malignancy of the nose, patients with previous history of nasal surgery were excluded as it was thought these could alter the findings of the study or confound to an extent. For this study, participants were recruited from the consulting rooms of the Ear Nose and Throat (ENT) clinic of the identified facility. Four out of six consulting room doctors recruited participants for the study. These doctors took the telephone contacts of their patients after they had informed them of the study. Through this approach, patients who were diagnosed with allergic rhinitis were purposively and conveniently sampled. The doctors had five (5) working days each in a week and their average chance of identifying clients that met the set criteria was one or no client because the condition was not seasonal at the time of data collection. Therefore, the total number of participants recruited during the period was 40 . This enabled researchers to employ professional judgment to select samples who will provide the needed information. At the end of the period, all 40 patients were purported to be used for the study. All participants were asked comprehensive, open-ended questions which were about their experiences of allergic rhinitis triggering asthmatic attacks. The interview explored the following areas, 1) Patient experience on their physical well-being, and 2) Effects of the symptoms on their psychological well-being. The interview guide consisted of three sections. Section I contained demographic information of participants such as age, gender, marital status, occupation and number of years they had been suffering from Allergic Rhinitis. Section II covered the clinical features experienced by the participants while section III asked questions on the effects of allergic rhinitis on their psychological wellbeing.

Data was collected by conducting face-to-face in-depth interviews with each participant using an interview guide with prompts. The interviews started right after the first patient was identified and he agreed to be part of the study. Participants were given codes in an alphabetical order. The principal investigator followed up with and arranged for a meeting on phone. The principal investigator and a research assistant met the patient/participant at an agreed upon venue and time. The objective and the purpose of the study were made clear to the patient and the patient signed a written consent form. This approach was repeated until patients had nothing more to say and/or a saturation level attained with no new issues evolving from the participants. This information was recorded by a recorder and with taking notes as memos. These memos were dated to easily correspond with the data collected. The researcher probed during the interview to focus on responses within the objectives of the study and get in-depth responses. The duration for all interviews ranged between 30 and 45 minutes. Each interview was transcribed verbatim and analysed at the end of each day. Participants were allowed to authenticate key issues at the end of each analyzed interview through phone calls to ensure the credibility of data generated. In all, fifteen (15) in-depth interviews were successfully conducted.

Data analysis occurred along side with data collection. This made it possible to explore emerging issues in successive interviews. Every interview was carefully listened too and transcribed verbatim from the local language into English by another member of the research team. The transcribed data was read through several times for familiarization, made notes and wrote down impressions of the transcripts. The data analysis was the matic using content analytic approach to analyze and manage the data obtained on the experiences of patients on their condition. Meaningful themes and sub- 
themes were generated. Quotes from participants were used to support subthemes in reporting the findings of the study.

\section{Ethical consideration}

The study did not cause any physical harm since it was a simple and non-invasive procedure. Participation was voluntary. A verbal followed by written consent was obtained from each participant. Ethical approval was obtained from the Institutional Review Board (IRB), University of Cape Coast as well as from the Committee on Human Research, Publication and Ethics, School of Medical Sciences in Kumasi.

\section{Trust worthiness}

To ensure trustworthiness of the study without sacrificing relevance, samples of the interview guide were given to colleagues for their input, after the guide was amended, it was given to members of faculty with requisite knowledge in the field of allergies to review before it was used.

\section{Results}

The study came out with three themes and two subthemes.

\section{Background characteristic of respondents}

All participants were diagnosed with allergic rhinitis at the Ear, Nose and Throat unit of this hospital. Table 1 provides a summary of participants' background information. Study participants aged between 23 and 58 years cutting across different spectrum of social class. There were fifteen (15) participants, four males and eleven females. The marital status of participants included: married $(n=7)$, unmarried $(n=7)$ and widow $(n=1)$. Years of experiencing allergic rhinitis span from four (4) months to 24 years. There was a range of educational level, including basic $(n=3)$, middle school $(n=2)$, secondary $(n=6)$, and tertiary $(n=4)$. Two interviews were conducted at the ENT clinic; eleven interviews were conducted at participants' places of work? Break time? and two were conducted at participants' home. All the participants resided in urban settlements within the Metropolis and the language of communication was English and the local dialect.

\section{Physical wellbeing of patient with allergic rhinitis}

This theme addressed the wellbeing of patients with allergic rhinitis. Participants were asked to tell how they experience allergic rhinitis. Three (3) subthemes were identified and they were the triggers, signs and symptoms and management strategies based on their views. The participants said they went through their signs and symptoms as a consequence of the allergy triggers. Therefore, they resorted to different management strategies to gain relief from the symptoms they experienced.

Allergytriggers referto participant'sconceptualization and definition of what sets their experience of allergic rhinitis in motion. Though, participants shared varying factors that led to the onset of the reaction, most of them identified dusty environment, use off an, cold environment, foul stench, and scented perfumes.

\section{This is reflected in the following response}

'What triggers these symptoms is when I get into contact with dusty environments, and since I am a seamstress, I may keep customers cloth at a place for a very long time and when I am about to work on them, it triggers these symptoms. Due to the stuffy nature and dust collected on the cloths in my container. Also,

Table 1: Background characteristics of participants.

\begin{tabular}{|c|c|c|c|c|c|}
\hline Participant & Age & Gender & Marital Status & Employment & Years of Experiencing AR/Years \\
\hline 1 & 40 & Female & Unmarried & Seamstress & 24 \\
\hline 2 & 39 & Female & Married & Seamstress & 12 \\
\hline 3 & 23 & Female & Unmarried & Unemployed & 1 \\
\hline 4 & 58 & Female & Widow & Enrolled Nurse & 39 \\
\hline 5 & 25 & Male & Unmarried & General Nurse & 3 \\
\hline 6 & 38 & Female & Married & Healthcare assistant & 12 \\
\hline 7 & 57 & Female & Married & Trader & 24 \\
\hline 8 & 41 & Female & Unmarried & Trader & 5 \\
\hline 9 & 32 & Female & Married & Midwife & 8 \\
\hline 10 & 24 & Female & Unmarried & Seamstress & 6 \\
\hline 11 & 47 & Female & Married & Teacher & 18 \\
\hline 12 & 44 & Male & Married & Steel Bender & 10 \\
\hline 13 & 37 & Male & Unmarried & Tailor & 1 \\
\hline 14 & 26 & Female & Unmarried & Student & 7 \\
\hline 15 & 36 & Male & Married & Trader & 8 \\
\hline
\end{tabular}

Field data 2019. 
at times, the nature of some clothes give out some kind of particles when trying to cut them into suitable dimensions to stitch them up. When I come into contact with these particles it triggers the symptoms like running nose. Sometimes smoke from the burning of rubbish at the public toilet behind my container, the fan I use in the container triggers the reaction. Participant 1 (Female)

Other participants narrated their experiences as.....

'What usually triggers these symptoms are when I blend pepper (chilli), I tend to sneeze and experience pain in my throat. Moreover, where my work is situated, there is a pub with a urinal just behind my shop, people use the urinal and I inhale this offensive smell of urine'.

\section{Participant 2 (Female)}

'Whenever I come into contact with smoke from the incinerator, aroma of burnt stew/sauce, burning of pepper, taking cold drinks and highly scented soaps, powder and perfumes, the symptoms come severely.

\section{Participant 7 (Female)}

Few of the participants said, eating of particular type of food predisposed or triggered the onset of rhinitis. Normally when I chew or take in groundnut (peanut) whether roasted or boiled. Also, when I take in the foreign mangoes too but the local ones do not trigger the symptoms. Participant 11 (Female)

\section{Clinical signs and symptoms experienced by participants}

These signs and symptoms border on what participants explained or suggested they observe or feel when they were suffering from allergic rhinitis, and some of these symptoms experienced by participants intensifies at night. These signs and symptoms stated by participants were the commonality and variedness in the diverse experiences. Principally, all participants identified and explained allergic rhinitis to be any condition experienced or suffered continuously characterized by or with one or all of the following; itching eyes, itching throat and ears, excessive sneezing, runny nose, blocked nose, headache, and pain in the throat, coughing, hawking and post nasal drip. These features defined the condition irrespective of what triggered it or where it was triggered. Confirming their experience with some participants shared the following...

I do experience itchy eyes, throat and ears and I do sneeze excessively; I do even urinate on myself during coughing. At times too, I do get runny nose with blocked nostrils which make breathing difficult for me. I have offensive breath too. Participant 1 (Female)

I do experience excessive sneezing, cough, reddened eyes, itchy palm and lips (even the whole body), urticarial rashes. Itchy eyes and sneezing with nasal discharge bother me a lot. I can even sneeze in a sense that I have to use my dress to even wipe the discharge (phlegms) that comes out of the sneeze. Participant 4 (Female)
I hawk whenever I experience this itchy throat, I didn't notice this hawking myself but I was told I do the hawking whenever I am asleep and rub my eyes and ears continually. Participant 10 (Female)

Among some participants, they experienced pains associated with the signs and symptoms such that it became irresistible leading to breath cessation and tearing.

'I experience cold, itchiness in my ear and throat. I get blocky nose and difficulty in breathing and tears usually drop'. Participant 6 (Female)

A similar experience was shared by a female health practitioner

'What I do experience is, I sneeze continuously which results in runny nose. When this symptom shows up, in the evening or night I'm unable (struggle) to sleep and even if I try to sleep, in about 2-3 hours, I will be awake. Because of these symptoms I'm always with a handkerchief'. Participant 9

Also, the onset of allergic rhinitis was confirmed to be all time round. However, validating responses from almost all the participants pointed to increased intensity of the symptoms during the night. Some participants shared;

'.... The condition is really disturbing especially when it gets to the night, you can't even sleep, and it gives you dry nose. One night I bathed with cold water because I was unable to sleep'. Participant 13 (Male)

The aggravation of the allergy during the nocturnal periods was characterized by blocked nostrils among some participants.

'When I try sleeping my nostrils tends to block and I have to come out from bed to stand or sit in fresh air for a while. At times I use pillows to position myself in order to breathe well'. Participant 14 (Female)

\section{Adopted home management strategies}

Concerning home management strategies, participants appeared to have learnt two principal approaches to dealing with the allergic rhinitis or condition. These had to do with immediate response to the allergy onset and a much-extended approach that took on the likeness of a coping strategy. In terms of the immediate management options, many participants had learnt the art of moving along with ample tissue or handkerchiefs and taking in warm fluids or bathing hot water while others coped with the condition through the use medication. The immediate medication was often over the counter drugs until the allergy severity necessitated hospital visits where participants were prescribed higher level medication.

'When I experience this runny nose, I do have tissues available with me that I use to blow my nose or wipe off the phlegm that comes out of my nose. When it reduces 
or stops, I use hand sanitizer or wash my hands under running water to disinfect my hands and proceed to what I was initially doing'. Participant 1 (Female)

Another participant explained, '...because of these symptoms I'm always with handkerchief.' Participant 9 (Female)

Taking in warm fluids, bathing hot water and application of warm compresses on the face were immediate remedy participants employ to relieve the symptoms. Participants narrated that...........

From my experience when the sneezing starts you can fart or urinate on yourself involuntarily. You feel like there's something choking your nose, so in case I feel that kind of symptoms, I boil warm water and place in a towel, squeeze the towel and place on my face. Participant 4 (Female)

'When my nose is blocked, I boil water, put in towel, squeeze it and place it on my nose'. Participant 8 (Female)

Normally when these symptoms show up, I drink lukewarm water or inhale the vapor of hot water. When I take in medications the symptoms suppresses'. Participant 6 (Female)

Changing of sleeping position was cited as an immediate management approach with the onset of the allergy.

'.....At times I use pillows to position myself in order to breathe well. I do experience this more than half of the days within the month'. Participant 14 (Female)

Supporting those who often used over the counter medication, a female student affirmed buying nasal spray and congestal tablets.

'....some time ago I was using avamys (nasal spray) and anytime I used it I experienced some tickly feeling and sneezed as well, so because of that I have stopped using it; it is quite expensive too. So, now I buy congestal tab for common cold which I take it when I am about to sleep in the evening'. Participant 14 (Female)

Failure by over-the-counter medication to relieve participants of the painful onset and continuous nasal discharge informed majority of the participants to seek medical attention. A participant indicated that......

'I tried numerous drugs but it still persisted so I went to the hospital and was prescribed some drugs which minimized the symptoms. The drugs got finished and in about two weeks I started experiencing the symptoms again. So, I sat down one day and decided to go to the referral site for treatment. When I went, they gave me drugs and told me to report in 1-week's' time, exactly the one-week time I went for review and the doctor told me it was improving and I should return in 2 weeks' time. I didn't show up any more at the hospital after the 2 weeks'. Participant 13 (Male)
Some participants while upholding referral advice on the utility of traditional or complementary medication on easing their suffering from the allergy, accessed traditional herbs to reduce the painful onset. Accordingly, the traditional herbal medication appeared to be efficacious though temporal in lessening the nasal discharge. A distinction was however made between herbal medicine use and visiting a herbal doctor. Participants claimed the latter did not offer any respite.

'For that matter people recommend some herbalist to me who they said she was powerful but it didn't resolve. I stopped using perfume and taking in cold water. ...... Because of the disturbing nature of the condition, I was directed to a certain man who they say he was perfect in douching the nose of those with symptoms of mine, I went there to douche my nose with some herbal concoction but still the symptoms persisted. I also went to another man who also gave me some powdery herbs, this powdery herb is the drug I have stuck to for now and I think I'm improving on my condition'. Participant 13 (Male)

\section{Psychological effects of allergic rhinitis}

This theme explores the mental state and behaviour of the patient as a result of the burden of the symptoms presented by the patient. The internal and external psychological moods of study participants were apprehensive by the condition. From the views expressed by participants, issues derived included the embarrassment and public shyness due to nasal discharge, sleep disorder, labeling, anxiety and discomfort, strained family relationship and poor concentration.

Regarding embarrassment and public shyness due to nasal discharge, the frequent phlegm release coupled with offensiveness of the breath continued to pose a psychological problem for some participants. This was affirmed among some participants as expressed below;

I do find it difficult in sleeping and very embarrassed. When I was growing up into adulthood, one thing I realized was, I feel like some offensive smells comes out from my nose and psychologically, I felt that people around me also perceived the offensive smell. I once asked my mom whether she can smell that offensive odour when she came closer to me, and she said no. But one thing my mom said was that whenever I slept, she does smell some offensive smell from my breathing. At times too when I spit out phlegm, I do see some creamy spots in the phlegm which has very bad smell. Participant 1 (Female)

Another participant explained that....

I always feel very embarrassed when colleagues tell me like "you are always with handkerchief and your headache is chronic". If you don't take it cool with their comments you may even get angry and quarrel with them. Participant 9 (Female) 
In relation to sleep disorder, there were those who had accompanying sleeping difficulties due to allergic rhinitis. This was coupled with smell differentiation, constant spiting and among some participants an unsightly frequent discharge of phlegm. A participant indicated sleeping as a major challenge with allergic rhinitis.

I cannot sleep because of sneezing and the blocked nose. Sleeping is my major problem because it affects me during the day'. Participant 4 (Female)

Other participants emphasized that....

'I do experienced difficulty in sleeping too so someone recommended a drug to me but whenever I stop or the drug gets finish the symptoms shows up again'. Participant 13 (Male)

With anxiety, while many considered the embarrassment to their person, others were worried about the effect the continuous discharge of phlegm had on the public when hawking in order to clear the throat.

'In public you need to hawk and it doesn't look pleasant and it feels like you are disturbing others with sneeze and itching throat. I am always with tissues just because of the sneezing'. Participant 14 (Female)

Another emphasized that.....

'I do think a lot about this condition, my brother once said the way I react and behave at night because of this condition when I marry on the day of honeymoon my husband will be scared and run away from the room'.

\section{Participant 10 (Female)}

Not only does the condition put study participants in an embarrassed and uncomfortable position, it also affected their involvement in social activities to avert the possibility of having to manage the condition in public when all attention might be on them. Among those categories of participants, a worried student explained;

'... I feel embarrassed when experiencing these symptoms in the midst of people. Also, when I am studying and I feel these symptoms. I always say I have a bad nose. I am very anxious about the condition...... I am still a student so I always think of it that when I start practicing as a medical doctor, will I be sneezing, coughing whiles I am to see a patients? So, I am just praying by the time I start practicing the condition won't be there anymore. Participant 14 (Female)

While some participants had considered the allergic condition as their way of life, others were worried about their labeling that was associated with the experience. In some instances, participants had come to be identified as those who are known for frequent cough and sneeze. This was narrated by a participant as;

'It is like everyday you are having cold and cough.
At Senior High School (SHS) all the teachers knew me because of my condition (cough and sneezing). Even at SHS one of my teachers said I will sneeze and my nose will fall off for a dog to bite in to it'. Participant 14 (Female)

Other participants stated that....

'Especially during my days as an apprentice, my colleagues hated to sit by my me when they are eating okra soup because of my runny nose. My colleagues always accused me of intentionally blowing my nose'. Participant 10 (Female)

I'm uncomfortable with this condition, and I'm unable to smell the aroma of foods. I always feel like I "talk in my head" (sound nasal). Even at work place everyone complains that I'm always having headache and always with handkerchief'. Participant 9 (Female)

Also, many of the participants indicated the discomfort they experience from the signs and symptoms. Similarly, as a coping strategy, most of the participants had to put their finger in their ears to experience some relief. This was considered very discomforting for many participants particularly those whose working conditions involved daily and constant engagement with clients. But tressing this dimension of discomfort, a participant narrated

'The condition has affected me, as I said you will put your finger in your ears just to get some relief from the symptoms unawares especially when you are in front of students teaching. I'm uncomfortable and frankly speaking I don't work as I want to. Naturally, I'm someone who is hardworking but when the symptoms build up, I'm unable to teach or work when I come to school'. Participant 11 (Female)

Participant's inability to perform their assigned roles and responsibilities due to the condition occasionally caused strained relations among siblings. This stemmed from another person having to perform roles assigned to study participants for which their inability to execute demands that others played those roles on their behalf. Some participants indicated;

'On Saturdays at home, I don't sweep or mop so my sisters take over and my sisters always quarrel with me for me not working because of my condition. My parents sometimes also accuse me of using my condition as an excuse not to work'. Participant 14

Likewise, participants indicated that the symptoms they experience enhanced poor concentration when performing daily activities. The reason being that their attention is most of the time on the symptoms and not the activity; hence it takes longer time in performing that activity. Views of participants on poor concentration included;

Because of these symptoms I'm always with 
handkerchief. Even at work place some of my duties that I can use few minutes to complete; I will spend a lot of time. I lose concentration when performing at my work place because all my attention is on the condition; so, rendering my productivity very low. Even the work that I can take 2 mins to do, I take more time to do because of poor concentration. Participant 9 (Female)

I'm not able to concentrate on my job, for instance when I experienced symptoms like runny nose, itchy eyes, I need to hold on, and get some relief before I can continue'. Participant 1 (Female)

\section{Discussion}

In terms of physical wellbeing, the patient with allergic rhinitis reported a burden of symptoms. These include excessive sneezing, runny nose, blocked nose, headache and pain in the throat, coughing, hawking, itching in the eyes, ears, nose and throat, fatigue, offensive breath, inability to smell, and lacrimation. Findings from this study supports similar findings reported by [12] in a study conducted in six countries across North America and Europe where majority of the respondents in all countries reported sneezing and itchy or watery eyes to be their most common perceived symptoms.

Also [13], reported that usually adults with allergic rhinitis are certainly troubled by the symptoms such as nasal blockage, rhinorrhoea and sneezing. They are mainly worried by continually having to carry paper tissues, blow their noses frequently and experiencing the urge to rub both nose and eyes. They also experience non-nasal symptoms that are troublesome such as thirst, itching of the palate and headache [13].

Moreover, a study conducted by [14] who confirms that AR causes upper airway symptoms such as nasal congestion, rhinorrhoea, and nasal itching and depending on the sensitivity of the allergen and persistent irritation of the upper airways; it may lead to chronic rhinitis and laryngitis. Hence the signs of laryngeal irritation and mucous production which is usually thick and sticky can dampen the vocal cords and consequently, oedema and excessive mucous on the vocal cords can negatively influence the voice quality and so, patients cough and clear their throats more frequently. The document suggested that patients should always consult the specialist for appropriate management of these signs and symptoms to avert any complications.

Furthermore, participants revealed that they go through these symptoms anytime they are exposed to triggers in the environment such as cold environment, inhalation of smoke, scented items, sitting under a fan, and offensive smell from the environment. They also reported triggers such as stuffy environment, dust, food and particles from clothes. This is supported by a study conducted by [15] who indicated that the most common triggers were dust, strong perfume odours and cold weather. However, participants from this study indicated other triggers such as that were not reported by their study.

In addition to their physical wellbeing, participants reported that they usually self-managed the condition before seeking for specialized intervention. Such management strategies included, avoiding the triggers, steam inhalation, taking warm fluids, use of over-thecounter medication and use of herbal preparations. Most of the participants reported to the hospital when the condition was severe and they were burdened with the symptoms. Similar findings were reported by [16] where patients diagnosed with allergic rhinitis seem to be self-managing their condition and interact less with their doctor about allergy prescription. Such patients interacted with the pharmacist about their allergy medication more commonly than interacting with the physician. Another survey by [17] cautions that persons with rhinitis who do not consult a physician when symptomatic will report multiple sensitizations, sleep disturbances and cognition disturbances. Also, drug utilization among persons reporting to the pharmacy with moderate or severe symptoms seems to be substandard. Thus, reliance on pharmacy or selfmedication may not be suitable for individuals with allergic rhinitis.

If allergic rhinitis is not properly controlled, it can predispose the individual to rhino-sinusitis, otitis media, nasal polyps, allergic conjunctivitis and hearing loss. Hence early diagnosis and management of allergic rhinitis promotes health $[12,18,19]$. Regarding the psychological effects, the participants reported various effects. Most participants reported that they always had to clear their throat, blow the nose and clean the nasal discharge which is an embarrassment to them especially when they had to do these in public. In addition, most participants indicated that they do experience sleep disturbances due to the blocked nose as it impairs their breathing, hence their inability to sleep at night and rather feel sleepy during the day.

\section{Quality of Life}

Some participants also reported that the condition is worrisome and so they feel uncomfortable and depressed. Consequently, they experienced emotional disturbances such as anxiety and mood changes. The study results also indicated that the participants had poor concentration during the performance of any activity as their attention was always on the symptoms they experience. These findings supported [20], that sleep is fundamental for physical and psychological health, patients with chronic diseases, including chronic respiratory diseases, usually have considerably impaired sleep quality that may increase the frequency of exacerbations and severity of symptoms, leading to difficulty in patient management, and reducing Quality Of Life (QoL) [20]. Also reported that the 
effect of rhinitis on a patient goes beyond specific anterior nasal symptoms. Nasal obstruction can cause sleep disturbances that reduce a patient's day time concentration and lead today time sleepiness.

Furthermore, a cross-sectional epidemiological study conducted by [21] reported that the impact of the severity of rhinitis was significant for insomnia, severe insomnia, hypersomnia, respiratory arrest, observed apnoea, sleepiness, and regular use of sedatives. Thus, poorly controlled symptoms of allergic rhinitis may also contribute to sleep loss or disturbances, secondary daytime fatigue, and decreased overall cognitive functioning. Also, a study conducted in Korea reported that AR patients were at higher risk of stress and depressed mood and required more psychological consultations [22]. Likewise, persistent and severe AR was associated with poor mental health [23]. Reported that AR had more negative effect on perceived stress and depressed mood. Findings such as labeling and strained family relationships were not indicated in literature. It is therefore important for individuals diagnosed of allergic rhinitis not to under estimate the condition as it can have adverse effect on their Quality of Life.

\section{Conclusion}

Allergic Rhinitis is a significant health problem, yet a neglected disorder especially in Ghana. It is often under recognized and underreported. Patients diagnosed with allergic rhinitis experience a wide range of troublesome signs and symptoms which affects their quality of life negatively. This study revealed that in the two dimensions of quality of life (physical and psychological), participants experienced several symptoms as they got exposed to allergy triggers resulting in poor concentration, sleep disturbances, and emotional disturbances. Moreover, allergy triggers were from the patients' environment which they need to avoid or protect themselves to minimize the frequency of the condition. Though all participants reported to the specialist for medical intervention, most of them still use over-the-counter medication whenever they experience the symptoms instead of reporting to the hospital. Findings such as labeling and strained family relationships calls for psychosocial counselling/support for people suffering from allergic rhinitis.

\section{Strengths and Weaknesses of the Study}

The transferability of the findings of this study is limited as the quality of life in only two dimensions is subjective and may differ from patient to patient and/or the kind of allergen triggers in the environment. Since a qualitative method was employed, the findings cannot be generalized because few allergic rhinitis patients were interviewed.

\section{Ethical Approval}

A gatekeeper letter from IRB with an introductory letter from the school of nursing was added to the request to the research and development unit of the hospital. Ethical clearance from the hospital was also obtained from the School of Medical Sciences of the Hospital Committee on Human Research, Publication and Ethics for permission to conduct the study at the designated area before the interview was done.

\section{Nursing Implication}

With reference to the findings of this study the nursing implication could be that, practitioners need to acknowledge the existence of the condition. There is the need to intensify health education through outreach services in schools, churches and use of mass media to create awareness of this trivialized and neglected condition. There should be better communication between practitioners and patients to give them understanding of this disorder.

\section{Funding}

There was no funding for this study.

\section{Competing Interests}

The authors declare that they have no competing interests.

\section{Authors' Contributions}

MBB conceived the study, was the principal investigator and made most extensive contribution to the research. AAD and RHR were involved in the conception of the research, guided the development of the proposal and supervised the study. IDM and AK identified the patients used for the study. IKA and PW revised the research and developed a manuscript for its intellectual and professional content. IDM and AK concluded diagnosis of the patients and helped with recruitment.

\section{Acknowledgements}

We thank the hospital that granted permission to conduct this research, and the ENT nurses who agreed to allow their space to be used for the study and the patients for the time spent in the interviews.

\section{Data Availability Statement}

Data will be availability to any researcher upon reasonable request.

\section{References}

1. Bozek A (2017) Pharmacological management of allergic rhinitis in the elderly. Drugs Aging 34: 21-28.

2. Al Suleimani YM, Walker MJA (2007) Allergic rhinitis and its pharmacology. Pharmacol Ther 114: 233-260.

3. Mahboub B, Al-Hammadi S, Prakash VP, Sulaiman N, Blaiss MS, et al. (2014) Prevalence and triggers of allergic rhinitis in the United Arab Emirates. World Allergy Organ J 7: 19.

4. Gryglas A (2016) Allergic rhinitis and chronic daily headaches: Is there a link? Curr Neurol Neurosci Rep 16: 33. 
5. Dhong HJ (2013) Classification of allergic rhinitis: What is most suitable in Korea? Allergy Asthma Immunol Res 5: 65-67.

6. Frati F, Dell'Albani I, Passalacqua G, Bonini, S, Rossi O, et al. (2014) A survey of clinical features of allergic rhinitis in adults. Med Sci Monit 20: 2151-2156.

7. Zheng $\mathrm{H}$, Dietrich $\mathrm{C}$, Thompson, $\mathrm{CL}$, Meuser $\mathrm{K}$, Brune A (2015) Population structure of endomicrobia in single host cells of termite gut flagellates (Trichonympha spp.). Microbes Environ 30: 92-98.

8. Green RJ, Davis G, Price D (2007) Concerns of patients with allergic rhinitis: The allergic rhinitis care programme in South Africa. Prim Care Respir J 16: 299-303.

9. Ozdoganoglu T, Songu M, Inancli HM (2012) Quality of life in allergic rhinitis. Ther Adv Respir Dis 6: 25-39.

10. Antolín-Amerigo D, Tabar IA, del Mar Fernández-Nieto M, Callejo-Melgosa AM, Muñoz-Bellido FJ, et al. (2017) Satisfaction and quality of life of allergic patients following sublingual five-grass pollen tablet immunotherapy in Spain. Drugs Context 6: 212309

11. Dziekanski M, de Freitas Marcelino T (2017) Quality of life in pediatric patients with allergic rhinitis treated at the medical clinic of integrated education - Unisul. Int Arch Otorhinolaryngol 21: 371-376.

12. Canonica GW, Mullol J, Pradalier A, Didier A (2008) Patient perceptions of allergic rhinitis and quality of life: Findings from a survey conducted in Europe and the United States. World Allergy Organ J 1: 138-144.

13. Juniper EF (2006) Rhinitis quality of life in clinical practice. THE UCB Institute of Allergy.

14. Develioglu ON, Paltura C, Koleli H, Kulekci M (2013) The effect of medical treatment on voice quality in allergic rhinitis. Indian J Otolaryngol Head Neck Surg 65: 426-430.
15. Said SA, Mchembe MD, Chalya PL, Rambau P, Gilyoma JM (2012) Allergic rhinitis and its associated co-morbidities at Bugando Medical Centre in Northwestern Tanzania; A prospective review of 190 Cases. BMC Ear Nose Throat Disord 12: 13.

16. Kuehl BL, Abdulnour S, O'Dell M, Kyle TK (2015) Understanding the role of the healthcare professional in patient self-management of allergic rhinitis. SAGE Open Med 3: 2050312115595822.

17. Williams A, Scadding $G$ (2009) Is reliance on self-medication and pharmacy care adequate for rhinitis patients? Int $\mathrm{J}$ Clin Pract 63: 98-104.

18. Mir E, Panjabi C, Shah A (2012) Impact of allergic rhinitis in school going children. Asia Pac Allergy 2: 93-100.

19. Padjas A, Kehar R, Aleem S, Mejza F, Bousquet J, et al. (2014) Methodological rigor and reporting of clinical practice guidelines in patients with allergic rhinitis: QuGAR study. J Allergy Clin Immunol 133: 777.e4-783.e4.

20. Muliol J, Maurer M, Bousquet J (2008) Sleep and allergic rhinitis. J Investig Allergol Clin Immunol 18: 415-419.

21. Annesi-Maesano I, Hulin M, Lavaud F, Raherison C, Kopferschmitt C, et al. (2012) Poor air quality in classrooms related to asthma and rhinitis in primary schoolchildren of the French 6 cities study. Thorax 67: 682-688.

22. Kim YS, Kim NH, Seong SY, Kim KR, Lee GB, et al. (2011) Prevalence and risk factors of chronic rhinosinusitis in Korea. Am J Rhinol Allergy 25: 117-121.

23. Shin JH, Roh D, Lee DH, Kim SW, Kim SW, et al. (2018) Allergic rhinitis and rhinosinusitis synergistically compromise the mental health and health-related quality of life of Korean adults: A nationwide population-based survey. PloS One 13: e0191115. 